\title{
HYPOKALEMIC PERIODIC PARALYSIS ASSOCIATED WITH TUBULOINTERSTITIAL NEPHRITIS IN A PATIENT WITH SJÖGREN'S SYNDROME
}

Danielly Dantas Pimentel ${ }^{1, \star}$, Gizelle Gouvea Rezende ${ }^{1}$, Gustavo Roberto Lourenço ${ }^{1}$, lane Tamara Dondé ${ }^{1}$, Juliana de Jesus Boscolo ${ }^{1}$, Luciana Akita ${ }^{1}$, Viviane Alves Costa ${ }^{1}$, Viviane Queiroz de Oliveira Maia ${ }^{1}$, Taisa Morete da Silva ${ }^{1}$

1.Faculdade de Medicina de São José do Rio Preto, São José do Rio Preto (SP), Brazil.

*Corresponding author: daniellydantas2011@gmail.com

\section{BACKGROUND}

Sjögren's syndrome (SS) is a systemic autoimmune disease characterized by chronic inflammation of the exocrine glands, mainly the salivary and lacrimal ones, causing keratoconjunctivitis sicca, xerostomia, xerotrachea and vaginal dryness. The population prevalence is $0.5-1 \%$ and it predominantly affects middle aged women in their fourth decade of life. Renal involvement can be found in up to $5 \%$ of patients with the primary form of the disease, occurring as interstitial nephritis or glomerulonephritis.

\section{CASE REPORT}

A 39-year-old female patient, with no previously known comorbidities. She reported two episodes of parotitis in 2006 and 2009 that were resolved with antibiotic treatment. In 2012, she was hospitalized with hypokalemic periodic paralysis. As of 2014, she started to follow up with the ophthalmology team due to xerophthalmia, at the time with a $2 \mathrm{~mm}$ Schirmer's test in the right eye and $3 \mathrm{~mm}$ in the left eye, with no defined etiology until then. In 2019, she underwent routine tests, which showed an increase in serum creatinine levels, hypokalemia and metabolic acidosis. Investigation of SS with distal tubular involvement was initiated. Salivary gland scintigraphy was performed, showing an important impairment in the accumulation and elimination of parotid and submandibular glands. Laboratory tests: potassium level of 3.3; creatinine level of 2.2; urine test showing hyposthenuria (urinary density of 1005) and urinary $\mathrm{pH}$ of 7; protein electrophoresis showing hypergammaglobulinemia; normal anion gap; chloride level of 107; positive rheumatoid factor; positive anti-Ro and anti-La; ANA: fine speckled nuclear pattern 1/640.

\section{CONCLUSION}

Type 1 renal tubular acidosis, also known as distal RTA (dRTA), is an impairment of renal tubule acidification due to a defect in the intercalated alpha cells of the cortical collecting duct that causes the H+-ATPase pumps to fail to secrete acid into the urine, resulting in hypokalemia, hyperchloremic metabolic acidosis and alkaline urinary pH (above 5.5). There is also hyposthenuria, due to interference with the urine-concentrating mechanism. Similar results were found in the reported patient's exams. Faced with the dRTA disease, Sjögren's syndrome is a diagnostic hypothesis to be considered. Patients with SS and dRTA can develop hypokalemic periodic paralysis in almost $40 \%$ of cases, which may serve as a marker of high-severity kidney disease. Hypokalemic paralysis may be the first manifestation of the disease, preceding the symptoms of xerostomia and xerophthalmia. The reported patient presented a similar condition, with later development of sicca syndrome and diagnosis of primary SS. 